OPEN ACCESS

Edited by:

Marco Tamietto,

Tilburg University, Netherlands

Reviewed by:

Frank A. Russo,

Ryerson University, Canada

Iris I. A. Groen,

New York University, United States

*Correspondence:

Luca F. Ticini

Iuca.ticini@manchester.ac.uk

Specialty section:

This article was submitted to

Emotion Science,

a section of the journal

Frontiers in Psychology

Received: 26 July 2017 Accepted: 17 November 2017 Published: 01 December 2017

Citation:

Ticini LF, Urgesi C and Kotz SA (2017) Modulating Mimetic Preference with Theta Burst Stimulation of the Inferior

Parietal Cortex.

Front. Psychol. 8:2101.

doi: 10.3389/fpsyg.2017.02101

\section{Modulating Mimetic Preference with Theta Burst Stimulation of the Inferior Parietal Cortex}

\author{
Luca F. Ticini ${ }^{1 *}$, Cosimo Urgesi ${ }^{2,3}$ and Sonja A. Kotz ${ }^{4,5}$ \\ ${ }^{1}$ Division of Neuroscience and Experimental Psychology, Faculty of Biological, Medical and Health Sciences, School of \\ Biological Sciences, University of Manchester, Manchester, United Kingdom, ${ }^{2}$ Department of Languages and Literatures, \\ Communication, Education and Society, University of Udine, Udine, Italy, ${ }^{3}$ Istituto di Ricovero e Cura a Carattere Scientifico \\ "Eugenio Medea", Bosisio Parini, Italy, ${ }^{4}$ Department of Neuropsychology, Max Planck Institute for Human Cognitive and Brain \\ Sciences, Leipzig, Germany, ${ }^{5}$ Department of Neuropsychology and Psychopharmacology, Faculty of Psychology and \\ Neuroscience, Maastricht University, Maastricht, Netherlands
}

We like an object more when we see someone else reaching for it. To what extent is action observation causally linked to object valuation? In this study, we set out to answer to this question by applying continuous theta burst stimulation (cTBS) over the left inferior parietal lobule (IPL). Previous studies pointed to this region as critical in the representation of others' actions and in tool manipulation. However, it is unclear to what extent IPL's involvement simply reflects action observation, rather than a casual role in objects' valuation. To clarify this issue, we measured cTBS-dependent modulations of participants' "mimetic preference ratings", i.e., the difference between the ratings of pairs of familiar objects that were (vs. were not) reached out for by other individuals. Our result shows that CTBS increased mimetic preference ratings for tools, when compared to a control condition without stimulation. This effect was selective for items that were reached for or manipulated by another individual, whilst it was not detected in non-tool objects. Although preliminary, this finding suggests that the automatic and covert simulation of an observed action, even when there is no intention to act on an object, influences explicit affective judgments for objects. This work supports embodied cognition theories by substantiating that our subjective preference is grounded in action.

Keywords: action, observation, preference, tools, inferior parietal lobule, cTBS, objects, affective judgments

\section{INTRODUCTION}

In the last decades, research has shown that people commonly prefer objects that are the goal of others' actions, regardless of the objects' actual value(s) or their intrinsic properties (Gollwitzer and Moskowitz, 1996). For instance, on the playground children run after the same toy even when similar toys are readily available. In social cognition, this behavior has been termed mimetic desire (Girard, 1988) and it is recognized as a case of goal contagion (Aarts et al., 2004), for which objects that are the target of another individual tend to become the goal for the observer. A functional neuroimaging study (Lebreton et al., 2012), designed to uncover the neural mechanisms of this phenomenon, surprisingly found that object preference is influenced by the activity of motor-related areas, namely the ventral premotor and the inferior parietal cortices, belonging to the action observation-action execution network (which is active during motor act execution as well as 
the observation of motor acts performed by others; Rizzolatti and Craighero, 2004; Fabbri-Destro and Rizzolatti, 2008; Avenanti and Urgesi, 2011). In particular, the authors found that during the observation of goal-directed actions the activity of these parietofrontal areas modulated that of the ventral valuation system where the perceived value of objects is encoded (Rangel et al., 2008; Chib et al., 2009; Lebreton et al., 2009; Peters and Büchel, 2010). However, as the correlational nature of neuroimaging cannot provide a direct causal link between brain and function, it is an open question whether this activity is merely an epiphenomenon of action perception or whether it truly reflects a neurocomputational process functionally relevant for the expression of subjective preference for a particular object. The present experiment represents a preliminary approach aimed at deciding between these two alternatives.

In healthy participants, we used offline continuous theta burst stimulation (cTBS) to interfere with the neural activity of the left inferior parietal lobule (IPL). Among the numerous TMS stimulation protocols available (for a review, see Fitzgerald et al., 2006), we used cTBS as it is known to induce, after a short and tolerable stimulation (40 s), prolonged neural inhibition (>45 min; Huang et al., 2005; Franca et al., 2006) at stimulated loci, enough to last throughout our experimental sessions. The neuronal mechanisms related to the inhibitory (or excitatory) effects of brain stimulation are not fully understood, and they vary according to the type of stimulation (i.e., frequency, intensity, number of pulses). For instance, in the case of theta burst stimulation, previous investigations reported that the application of brief bursts resulted in a transient increase in cortical facilitation (Huang and Rothwell, 2004) whilst prolonged stimulation had the opposite effect (Di Lazzaro et al., 2005; Huang et al., 2005).

We targeted the IPL for two main reasons. First, in their imaging study, Lebreton and colleagues found that its activation correlated with an object's likability and represented the first node involved in translating action observation into preference (Lebreton et al., 2012). Second, IPL's activity codes for the agent-object relationship (reviewed in Gentilucci and Volta, 2008; Fernandino and Iacoboni, 2010). In other words, the IPL holds a higher-order representation of the goal of an observed action independently of the effector (e.g., hand or mouth) used to achieve it (Jastorff et al., 2010). On the contrary, activity in the ventral premotor cortex (i.e., the other node of the action observation-action execution network involved in mimetic desires) appears more clustered around the effector (e.g., specific muscle, joint, and digit movements) performing a motor act (Jastorff et al., 2010). Stimulation of the ventral premotor cortex is also more distressing as it activates superficial nerves and muscles in the "temporalis" muscle fascia.

In addition, it is well-established that the IPL has a functional role as an interface between perceptual and motor information. Indeed, it is pivotal in visuomotor transformations (Goodale and Milner, 1992; Jeannerod et al., 1995) converting the intrinsic properties (size and shape) of an object into a pattern of goaldirected movements to reach it such as grasping (Blakemore and Sirigu, 2003; Fogassi and Luppino, 2005) and translating visual information into motor programs to achieve imitations (Mühlau et al., 2005; Molenberghs et al., 2009). Further neuroimaging (Buccino et al., 2001; Chong et al., 2008; Jastorff et al., 2010), TMS (Cattaneo et al., 2010; Jacquet and Avenanti, 2015; for a review see Avenanti et al., 2013b), and lesion studies (e.g., Buxbaum et al., 2005; Kalénine et al., 2010; for a review see Urgesi et al., 2014) showed that the IPL is implicated in the representation of goals of observed actions (Fogassi et al., 2005) and in the internal simulation of an observed motor act (i.e., motor resonance; Gallese et al., 1996; Grafton, 2009) thus demonstrating its pivotal role in goal contagion. TMS (e.g., Ishibashi et al., 2011) and neuroimaging (Kellenbach et al., 2003; Boronat et al., 2005; Canessa et al., 2008) findings implicated the left IPL also in storing motor representations for using familiar tools, in accordance with the fact that damage in this area determines tolluse deficits as shown by apraxic patients (Buxbaum et al., 2000; Rosci et al., 2003).

We chose to stimulate the left IPL for two reasons. First, because IPL's activity is generally stronger in the left hemisphere (see Peeters et al., 2009; Jastorff et al., 2010). Second, because in our experiment the majority of the observed motor acts were carried out with the contralateral right hand (see Methods; research has shown hemispheric preference for contralateral effector movements, see Pelphrey et al., 2004; Shmuelof and Zohary, 2006).

Grounding our experiment on the evidence listed above, we hypothesized that targeting the IPL with cTBS would demonstrate the casual involvement of action perception in an objects' preference. We therefore looked for variations in mimetic preference ratings (obtained comparing participants' ratings of objects that were, vs. were not, reached for by others) for familiar tools and non-tool objects, when cTBS was (or was not) applied. Our prediction was that cTBS would interfere with covert simulation of observed actions, particularly those carried out on tools, and in turn this would reduce mimetic preferences for these items. Such result would support the existence of a causal link between one's motor experience and preferences for non-valence stimuli (i.e., without an inherent emotional content). It would also support embodied cognition theories (for a review, see Ping et al., 2009) in social behavior (Aarts et al., 2004) as well as in aesthetics (Freedberg and Gallese, 2007; Cross et al., 2011; Cross and Ticini, 2012; Ticini et al., 2014) postulating that subjective preference is influenced by others' behavior.

\section{METHODS}

\section{Participants and Experimental Protocol}

This study was carried out in accordance with the recommendations of the Ethics committee of the Scientific Institute (IRCCS) Eugenio Medea with written informed consent from all subjects, in accordance with the Declaration of Helsinki. The experiment was carried out on 12 volunteers (aged $22.7 \pm$ 4.6 years; four females; 11 right-handed and one mixed-handed, Briggs and Nebes, 1975), complying with what according to Sack et al. (2009) is thought to be sufficient to demonstrate a behavioral effect in case of TMS based on group Talairach coordinates. 
We used 120 short videos (duration: $2-5$ s; $640 \times 480$ pixels) from Lebreton et al. (2012) categorized in familiar tools (e.g., pen, lantern, comb, soap bottle) and non-tool objects (e.g., food, toys such as cards and teddy bear, clothes, flowers). In 60 videos, the objects were reached for by another agent ("Goal-objects") who either moved them or not (Figure 1A). To remove the potential impact of eye gaze on the participant's judgment, the face of the agent was never visible (e.g., Bayliss et al., 2006). In $70 \%$ of the videos the action was executed with the right hand, in $6.7 \%$ with the left hand, in $15 \%$ with the mouth, and in $8.3 \%$ with other effectors (e.g., nose). The other 60 videos ("NoGoal-objects"; Figure 1B) depicted the same objects but this time they were presented either statically (48\% of the objects) or they were moved by a natural forces (e.g., wind or gravity with no human interaction; $20 \%$ of the objects) or a human agent was present but did not interact with the objects $(32 \%$ of the objects). This allowed us to control for the confound that participants may prefer objects in Goal-objects conditions because of the presence of a human being or because of some movement in the video. The two objects within a pair (Goaland NoGoal-objects) differed only by color and were otherwise identical. For instance, a green candy was the Goal-object for half the subjects, and the NoGoal-object for the other half. This allowed us to eliminate color preferences at the group level (cf. Lebreton et al., 2012).

Each trial started with a fixation cross $(1.5 \mathrm{~s})$ followed by one of the videos. After each video, the object reappeared $(436 \times 326$ pixels $)$ and participants were required to rate it ("How much do you like the object?") by moving a cursor (randomized initial position) on a sliding scale with their right hand (from 0 or not liked, to 10 or highly liked). The question was focused on the object because we aimed at assessing whether observing object-directed actions increased the perceived value of the object. Moreover, previous research demonstrated that the precise formulation of the question (e.g., "How much do you like to use the object?" or "How much would you like to acquire the object?") is not crucial (Lebreton et al., 2012). There were no time constraints and the video presentation (Goal-objects/NoGoalobjects, Tool/Non-tool stimuli) was randomized. Stimulus presentation was obtained by using the Cogent 2000 (Wellcome Trust Centre for NeuroImaging, London, UK) library of MatLab (Math-Works).

The experiment was run twice for a total of 240 videos. In one session (i.e., cTBS), participants were first positioned for the purpose of the experiment and then cTBS was administered (the experiment started immediately after cTBS). In the other (i.e., Non-cTBS), the TMS coil was never held against the participant's head. The order of the session was counterbalanced. Each of the two sessions lasted $10 \mathrm{~min}$ on average and both were run with the very same experimental setup and separated by an interval of $1 \mathrm{~h}$. We applied cTBS over the left IPL (MNI coordinates $-58,-32,44$; transformed in Talairach space for the use of the neuronavigation software SofTaxic Optic, E.M.S. s.r.l., Bologna, Italy) corresponding to the supramarginal and angular gyri as identified by Lebreton et al. (2012). cTBS consisted of trains of bursts of three pulses at $50 \mathrm{~Hz}$ repeated at $5 \mathrm{~Hz}$ and delivered uninterrupted for $40 \mathrm{~s}$ for a total of 600 pulses (Huang et al.,
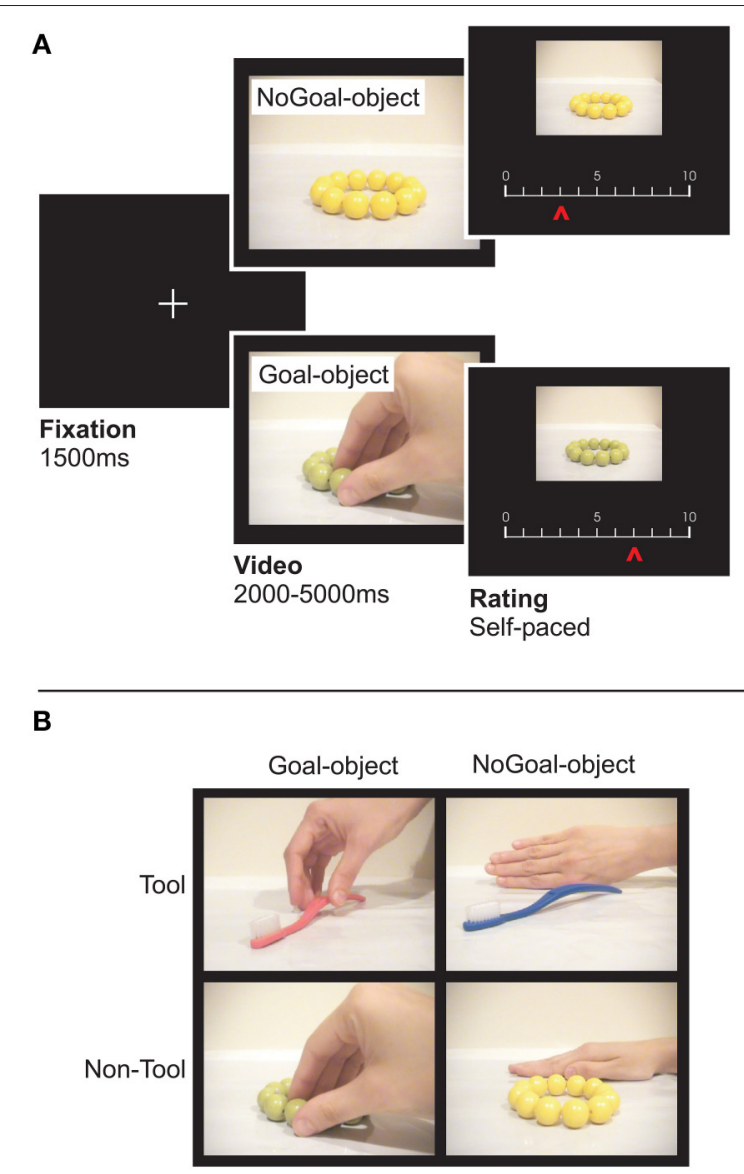

FIGURE 1 | The preference-rating task. (A) From left to right, the figure shows successive screens displayed in one trial. Participants rated the object ("How much do you like the object?") featured in the video by moving a cursor along a scale. Each trial started with a fixation cross followed by a video in which an item was (Goal-objects) or was not (NoGoal-objects) the target of another person's action. Then the preference scale appeared below the picture of the object to be rated (without a human agent). Two versions of identical objects, differently colored (counterbalanced across participants) were used to eliminate potential effects of color preferences at the group level.

(B) Snapshots from the videos with Toll and Non-Tool objects. In the illustrated example, in the NoGoal-object conditions, the objects were presented statically alongside a hand that did not interact with them. These and other control conditions (e.g., the object being moved by gravity) allowed avoiding the confound that participants may prefer objects more because of the presence of a human being or because of some movement in the video.

2005). It was delivered by an air-cooled figure-of-eight coil of $70 \mathrm{~mm}$ diameter attached to a Magstim Rapid2 stimulator, which was kept perpendicular to the underlying gyrus with the handle pointing upward and supported manually. The stimulation intensity was set at $45 \%$ of the maximal stimulator output (see for instance Stewart et al., 2001) for all participants. Exclusion criteria were the regular use of drugs or medications, any history of psychiatric or neurological disorders, and contraindications to transcranial magnetic stimulation (e.g., pregnancy or metallic implants), all assessed by a standard questionnaire. All participants wore earplugs and no particular discomfort or other negative side effects were reported. 


\section{Analysis}

We first conducted an omnibus repeated measured ANOVA, after having reduced the effect of inter-subject variability by normalizing ( $z$ scores) the preference ratings for each participants. We used "Stimulation" (cTBS, Non-cTBS) and "Action" (Goal, NoGoal-object) and "Object type" (Tool, Nontool) as within subjects factors. Additionally, we computed the mimetic preference ratings (see Lebreton et al., 2012) as the difference in the original ratings between Goal vs. NoGoal conditions (preference for Goal-objects-preference for NoGoalobjects). This excluded effects associated with variations in preference scores among objects, as we postulated that the objects were non-valenced stimuli (i.e., without an inherent emotional content). We then used one-sample Bayesian $t$-tests to test the hypothesis that the population means for the mimetic preference in the four conditions (cTBS and No-cTBS for Tools and Nontool objects) was not equal to zero. Bayesian $t$-tests (JASP; Love et al., 2015) employed default priors to estimate the Bayes Factors (BF; Rouder et al., 2012) and we used $\mathrm{BF}_{10}$ as it allows estimating the likelihood that the data fit a specified alternative hypothesis relative to the hypothesis of no effect (Rouder et al., 2012). $\mathrm{A} \mathrm{BF}_{10}$ greater than 3 indicates substantial evidence for the alternative hypothesis (Wetzels and Wagenmakers, 2012), i.e., that the observed data favor the alternative hypothesis over the null hypothesis by a ratio of 3:1.

\section{RESULTS}

The results of the ANOVA identified a statistically significant main effect of "Action" $\left[F_{(1,11)}=14.74, p=0.003\right.$, $\left.\eta_{p}^{2}=0.57\right]$ and a significant interaction between "Stimulation," "Action," and "Object type" $\left[F_{(1,11)}=5.1, p=0.045, \eta_{p}^{2}=0.32\right]$. Nonspecific effects of rTMS on the ratings ["Stimulation": $F_{(1,11)}=$ 1.3, $\left.p=0.28, \eta_{p}^{2}=0.1\right]$ and other factors or interaction ( $F \mathrm{~s}$ $<2.74$, ps $>0.12$ ) were absent. We then broke the ANOVA for the factor "Object type" and run selected tests comparing the effects of stimulation within each "Object type" category. For the Tools category, we found a significant main effect of "Action" $\left[F_{(1,11)}=23.55, p<0.001, \eta_{p}^{2}=0.68\right]$ and a significant interaction between "Stimulation" and "Action" $\left[F_{(1,11)}=7.9, p\right.$ $\left.=0.017, \eta_{p}^{2}=0.42\right]$. Bonferroni-corrected post-hoc tests showed that the preference for Goal-objects was larger $(p s<0.001)$ when compared to NoGoal-objects in both cTBS (Goal-objects: $0.108 \pm 0.054$; NoGoal-objects: $-0.211 \pm 0.040$; means \pm S.E) and Non-cTBS conditions (Goal-objects: $0.024 \pm 0.066$; NoGoalobjects: $-0.196 \pm 0.056$ ). Importantly, the preference scores between Goal-objects in cTBS were higher than those in NoncTBS $(p=0.036)$. This indicated that cTBS selectively increased the preference for Goal-objects rather than decreasing that for NoGoal-objects. Instead, the means for NoGoal-objects did not differ between the two stimulation conditions $(p>0.99)$. As far as the Non-tools category is concerned, we found a significant main effect of "Action" $\left[F_{(1,11)}=8.48, p=0.014, \eta_{p}^{2}=0.43\right]$ confirming that in both cTBS and Non-cTBS conditions Goalobjects were preferred more than NoGoal-objects. No other results were significant $(F s<2.8, p s>0.12)$. A further omnibus
ANOVA conducted on the judgements' response times revealed only a significant main effect of "Object type" $\left[F_{(1,11)}=5.17, p\right.$ $\left.=0.044, \eta_{p}^{2}=0.32\right]$ : the time required to express the preference for Tools (1968.38 $\pm 203.04 ; \mathrm{ms} \pm$ S.E.) was longer than that for Non-tools objects $(1931.97 \pm 155.20)$. Other main effects and interactions were not significant $(F s<1.96$, $p s>0.19)$.

Table 1 shows that all mean values of mimetic preference ratings (preference for Goal-objects-preference for NoGoalobjects) were significantly larger than the test value of zero (see Figure 2). This result was expected as previous experimental evidence (Lebreton et al., 2012) indicated higher preference for Goal-objects when compared to NoGoal-objects. Notably, the BF for Tools after cTBS was very large and indicated that the data favored the one-sided alternative hypothesis that the population mean was larger than the test value of zero by a ratio of 104.2: 1 . One-way ANOVAs calculated in each condition (i.e., Tools NoncTBS, Tools cTBS, etc.) indicated that the counterbalanced order of stimulation (i.e., whether participants begun the experiment with cTBS or Non-cTBS) did not influence the results $(F s<5.6$; ps $>0.16$ ).

A two-sample $t$-test (two-tailed) further showed that the mimetic preference for Tools after cTBS was significantly higher than that for Tools in the Non-cTBS conditions $\left[t_{(11)}=2.8, p=\right.$ $0.017, \mathrm{~d}=0.81$, C.I. 0.05 to 0.43 ]. The BF confirmed these results by indicating that the data favored the alternative hypothesis that the mimetic preference mean was higher in cTBS than in NoncTBS by a ratio of 3.8: 1 . This was clearly not the case for Non-tool objects $\left[t_{(11)}=-0.48, p=0.64, \mathrm{~d}=-0.14 ; \mathrm{BF}_{10}=0.3\right.$; C.I. -0.31 to 0.19$]$.

\section{DISCUSSION}

In this study, we used non-invasive brain stimulation to test the embodied cognition's hypothesis that subjective preference is grounded in action, while we recorded the preference of healthy volunteers for objects that were (or were not) reached for or manipulated by other individuals. The result indicates that IPL stimulation, which is known to interfere with processing goalrelated information (Cattaneo et al., 2010; Puzzo et al., 2013; for a review see Avenanti et al., 2013b) and tool manipulation (Ishibashi et al., 2011), led to a significant increase in mimetic preference ratings of tools. No effects were found on mimetic preferences of other familiar objects. We argue that, albeit preliminary, this result suggests a left IPL's contribution in integrating the representation of others' actions (Iacoboni et al., 1999; Grèzes et al., 2003; Johnson-Frey et al., 2003) with subjective preference for objects (Lebreton et al., 2009). We believe that the absence of an effect of the order of stimulation indicates that cTBS was not particularly disturbing per se (as people may rely more on others' choice in distressing situations) and did not induce arousal for objects (e.g., Dräger et al., 2004).

The present outcome prompts to question of why IPL's stimulation increased mimetic preference rather than the opposite, as we predicted. Indeed, previous research indicated that prolonged cTBS results in transient inhibitory effects (for a review, see Fitzgerald et al., 2006; Parkin et al., 2015). For 
TABLE 1 | Values of mimetic preference ratings measured across conditions.

\begin{tabular}{lccccccc}
\hline Conditions & Mean & S.E. & t-values & $\boldsymbol{p}$-values (two-tailed) & BF $_{\mathbf{1 0}}$ & C.I. & Cohen's d \\
\hline Tools Non-cTBS & 0.44 & 0.11 & 3.9 & 0.002 & $\mathbf{1 8 . 9}$ & 0.19 & 0.69 \\
Tools cTBS & 0.68 & 0.13 & 5.1 & $<0.001$ & $\mathbf{1 0 4 . 2}$ & 0.39 & 0.98 \\
Non-tools Non-cTBS & 0.53 & 0.16 & 3.3 & 0.008 & $\mathbf{7 . 4}$ & 0.17 & 0.88 \\
Non-tools cTBS & 0.47 & 0.13 & 3.6 & 0.004 & $\mathbf{1 2 . 6}$ & 0.19 & 0.76 \\
\hline
\end{tabular}

S.E., Standard error; BF, Bayes factor; C.I., Confidence interval.

The results of the statistical tests contrasting the means against the test value of zero are shown.
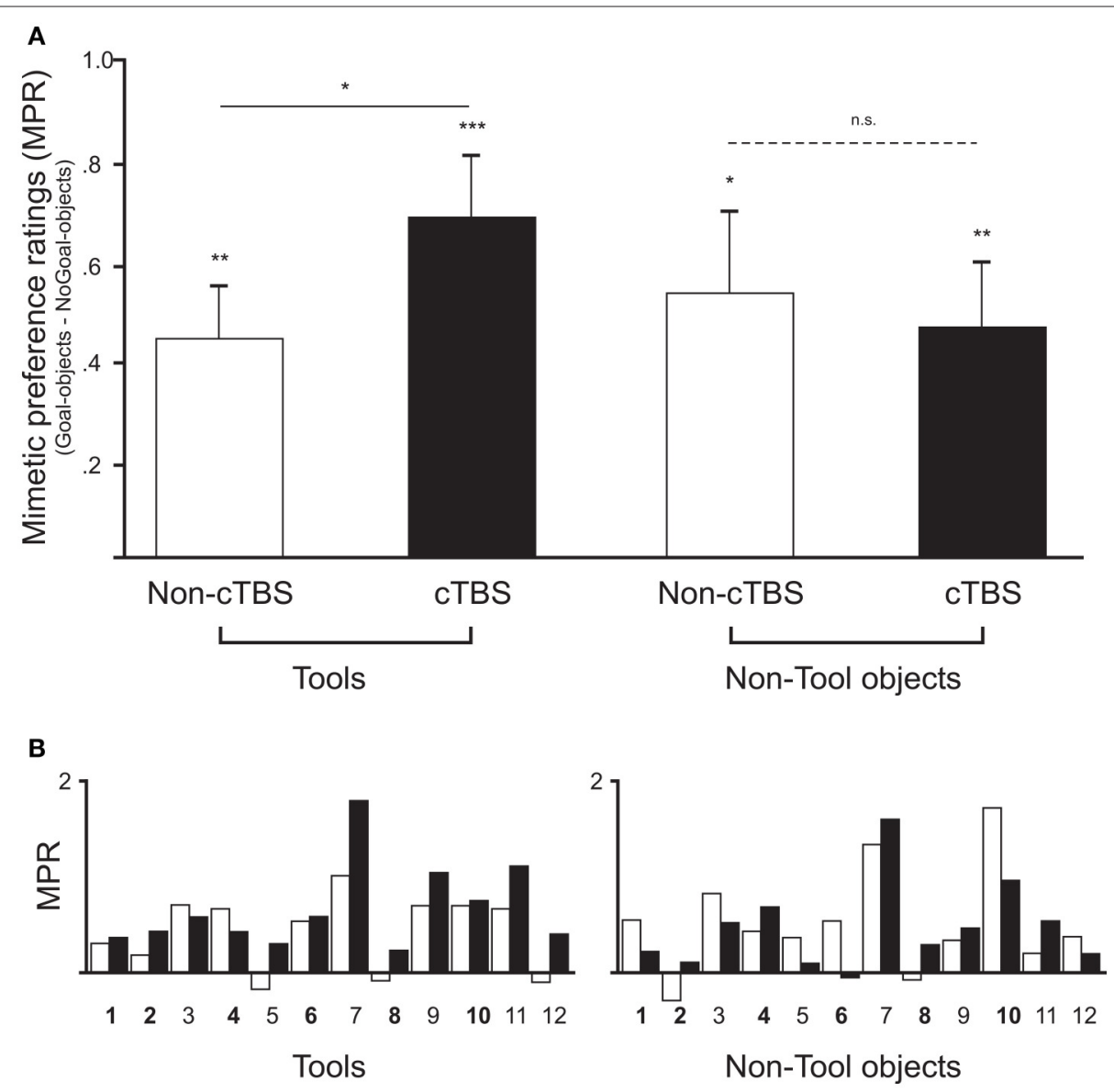

FIGURE 2 | (A) Comparison of mimetic preference ratings (difference in preference ratings between Goal- and NoGoal-objects) across participants for Tool and Non-Tools objects in the two stimulation conditions. Empty and filled bars represent mimetic preferences for the same objects in the Non-cTBS and cTBS session, respectively. Mean mimetic preference ratings were significantly positive in all conditions (means \pm standard errors of the mean; ${ }^{\star} p<0.05$, ${ }^{\star \star} p<0.01,{ }^{\star \star \star} p<0.001$ ). Two-sample $t$-tests (two-tailed) and Bayesian analyses indicated a statistically significant difference between Non-cTBS and cTBS in the Tools category (solid line), whereas no significant difference was found for Non-tool objects (dashed line). (B) Results for each individual (numbered along the X-axis) are shown along with the counterbalancing order of stimulation (in bold participants who received cTBS first).

instance, it determines a reduction in cortical excitability (or amplitude of motor-evoked potentials) when a testing single pulse of transcranial magnetic stimulation is applied over the primary motor cortex (Di Lazzaro et al., 2005; Huang et al., 2005). Although the mechanisms through which cTBS interferes with brain activity are not completely clear (Fitzgerald et al., 2006), some authors suggest that the change produced by cTBS may be related to a decreased effectiveness of synaptic connections recruited in the circuits that are involved in the generation of motor-evoked potentials (Huang et al., 2005).

In spite of the fact that some deemed irrelevant the direction of the behavioral effects when using TMS to empirically test the causal role of a brain area (Silvanto et al., 2008), two alternative explanations of our result are possible. On the one hand, cTBS may have increased the functional activation of the stimulated IPL (Siebner et al., 2001), thus permitting a better embodiment 
of the observed actions. In this regard, an interesting report indicated that interferential TMS over IPL improved recognition of emotional body movements (i.e., emotional expressions; Engelen et al., 2015). On the other, cTBS suppression of the IPL may have triggered compensatory activity in other sensorimotor areas with the result of facilitating the brain response to observed actions (e.g., Ubaldi et al., 2015). This apparently "paradoxical" facilitatory effect has been predicted and reported before (Kilner et al., 2007; Gazzola and Keysers, 2009; Friston et al., 2011; Schippers and Keysers, 2011; D'Ausilio et al., 2012; Arfeller et al., 2013; Avenanti et al., 2013a). In other words, disruptive cTBS over IPL may have increased motor resonance in frontal regions (Ubaldi et al., 2015) and in so doing facilitated the brain response to action observation (e.g., Avenanti et al., 2013a). In turn, as proposed by Lebreton et al. (2012), it is plausible that the increased activity in frontal areas would have engaged the ventral valuation system encoding the perceived value of objects (Rangel et al., 2008; Chib et al., 2009; Lebreton et al., 2009; Peters and Büchel, 2010). Obviously, the question of what are the underlying mechanisms associated to the spread of cTBS effects cannot be address in the current study as they would require, for instance, a combination of TMS and AMRI data in order to visualize compensatory processes. Another caveat (discussed below) is the absence of a control site. Overall, whether cTBS determined an increase or decrease of brain activity, our result seems to indicate that IPL belongs to a neural mechanism that translate the observation of goal-directed actions into preference ratings. We further believe that the pattern of our results rules out the possibility that the increase in mimetic preference toward tools was due to perceptual ease or fluency. This is observed when previous experience interacting with items affects one's own preference toward them (for a review, see Reber et al., 2004). Moreover, as the counterbalanced order of stimulation doesn't affect the pattern of results (see Results and Figure 2B), we can also exclude that our measurements were affected by behavioral facilitation. This is observed when the modulation of baseline neural activity though adaptation to a stimulus leads to statedependency TMS effects, i.e., the stimulation of functionally distinct neural populations within the targeted region (e.g., Cattaneo and Silvanto, 2008).

Additionally, we found that the time required to express the preference for tools was longer than for non-tool objects. This result could be explained, at least indirectly, by the fact that viewing tools automatically activates neural representations associated with their manipulation, which differ from that for other objects (Castiello, 2005; Proverbio et al., 2007, 2011, 2013; Cardellicchio et al., 2011). The integration of several complementary higher order relationships among action, affordance associated with tool manipulation, target and preference may require a longer processing time.

\section{REFERENCES}

Aarts, H., Gollwitzer, P. M., and Hassin, R. R. (2004). Goal contagion: perceiving is for pursuing. J. Pers. Soc. Psychol. 87, 23-37. doi: 10.1037/0022-3514.87.1.23
As mentioned above, a limitation of the current preliminary study is the absence of an active control stimulation site. The absence of control site means that we cannot exclude the possibility that cTBS applied elsewhere in the brain could cause similar effects on mimetic preference of tool objects. However, the choice of an "ideal" control site appears difficult when it comes to experiments requiring subjective judgements: TMS at different sites may influence participants' ratings in ways that cannot be always controlled for. For instance, TMS may cause diverse degrees of discomfort or distress associated with visual, acoustic, and tactile sensations or with activation of superficial nerves and muscles (e.g., when targeting the ventral premotor cortex through the "temporalis" muscle fascia). In addition, TMS of other sensorimotor areas (e.g., striate and extra-striate cortices, superior temporal sulcus) may interfere with the perception and processing of the visual stimuli per se (in our case, both Goaland NoGoal-objects), and stimulation of prefrontal and posterior parietal cortices may modulate object likability (e.g., Cattaneo et al., 2014) independently of whether an object is the goal of an action or not. Overall, we believe that the stimulation of the IPL was meaningful to address the research question, as demonstrated by the pattern of our results, and we acknowledge the fact that future studies should include an appropriate active control site.

To conclude, our preliminary result demonstrates that an object's preference is contingent on the representation of others' behavior in the brain of the beholder. We believe that this outcome is relevant to our understanding of the neural mechanism that, through actions, allows conveying the value of items from individual to individual and automatically influence preference choices for the objects present in our environment.

\section{AUTHOR CONTRIBUTIONS}

CU, LT, and SK conceived the work, interpreted the data, draft and revised the manuscript. LT and CU acquired the data and LT analyzed them.

\section{ACKNOWLEDGMENTS}

We wish to thank Mathias Pessiglione for his assistance with the stimuli and an earlier draft of the manuscript, and Marco Poliaghi for helping during data acquisition. The research was supported by a research fund from The University of Manchester and grants from the Ministero Istruzione Università e Ricerca, Italy (Futuro In Ricerca, FIR 2012, Prot. N. RBFR12F0BD; to CU) and from Istituto di Ricovero e Cura a Carattere Scientifico (Ricerca Corrente 2014; to $\mathrm{CU}$ ). The authors declare no competing financial interests. 
Avenanti, A., Annella, L., Candidi, M., Urgesi, C., and Aglioti, S. M. (2013a). Compensatory plasticity in the action observation network: virtual lesions of STS enhance anticipatory simulation of seen actions. Cereb. Cortex 23, 570-580. doi: 10.1093/cercor/bhs040

Avenanti, A., Candidi, M., and Urgesi, C. (2013b). Vicarious motor activation during action perception: beyond correlational evidence. Front. Hum. Neurosci. 7:185. doi: $10.3389 /$ fnhum.2013.00185

Avenanti, A., and Urgesi, C. (2011). Understanding "what" others do: mirror mechanisms play a crucial role in action perception. Soc. Cogn. Affect. Neurosci. 6, 257-259. doi: 10.1093/scan/nsr004

Bayliss, A. P., Paul, M. A., Cannon, P. R., and Tipper, S. P. (2006). Gaze cuing and affective judgments of objects: i like what you look at. Psychon. Bull. Rev. 13, 1061-1066. doi: 10.3758/BF03213926

Blakemore, S. J., and Sirigu, A. (2003). Action prediction in the cerebellum and in the parietal lobe. Exp. Brain Res. 153, 239-245. doi: 10.1007/s00221-003-1597-z

Boronat, C. B., Buxbaum, L. J., Coslett, H. B., Tang, K., Saffran, E. M., Kimberg, D. Y., et al. (2005). Distinctions between manipulation and function knowledge of objects: evidence from functional magnetic resonance imaging. Cogn. Brain Res. 23, 361-373. doi: 10.1016/j.cogbrainres.2004.11.001

Briggs, G. G., and Nebes, R. D. (1975). Patterns of hand preference in a student population. Cortex 11, 230-238. doi: 10.1016/S0010-9452(75)80005-0

Buccino, G., Binkofski, F., Fink, G. R., Fadiga, L., Fogassi, L., Gallese, V., et al. (2001). Action observation activates premotor and parietal areas in a somatotopic manner: an fMRI study. Eur. J. Neurosci. 13, 400-404. doi: 10.1046/j.1460-9568.2001.01385.x

Buxbaum, L. J., Johnson-Frey, S. H., and Bartlett-Williams, M. (2005). Deficient internal models for planning hand-object interactions in apraxia. Neuropsychologia 43, 917-929. doi: 10.1016/j.neuropsychologia.2004.09.006

Buxbaum, L. J., Veramonti, T., and Schwartz, M. F. (2000). Function and manipulation tool knowledge in apraxia: knowing 'what for' but not 'how'. Neurocase 6, 83-97. doi: 10.1080/13554790008402763

Canessa, N., Borgo, F., Cappa, S. F., Perani, D., Falini, A., Buccino, G., et al. (2008). The different neural correlates of action and functional knowledge in semantic memory: an fMRI study. Cereb. Cortex 18, 740-751. doi: $10.1093 /$ cercor/bhm110

Cardellicchio, P., Sinigaglia, C., and Costantini, M. (2011). The space of affordances: a TMS study. Neuropsychologia 49, 1369-1372. doi: 10.1016/j.neuropsychologia.2011.01.021

Castiello, U. (2005). The neuroscience of grasping. Nat. Rev. Neurosci. 6, 726-736. doi: $10.1038 / n r n 1744$

Cattaneo, L., Sandrini, M., and Schwarzbach, J. (2010). State-dependent TMS reveals a hierarchical representation of observed acts in the temporal, parietal, and premotor cortices. Cereb. Cortex 20, 2252-2258. doi: 10.1093/cercor/bhp291

Cattaneo, Z., Lega, C., Gardelli, C., Merabet, L. B., Cela-Conde, C. J., and Nadal, M. (2014). The role of prefrontal and parietal cortices in esthetic appreciation of representational and abstract art: a TMS study. Neuroimage 99, 443-450. doi: 10.1016/j.neuroimage.2014.05.037

Cattaneo, Z., and Silvanto, J. (2008). Investigating visual motion perception using the transcranial magnetic stimulation-adaptation paradigm. Neuroreport 19 , 1423-1427. doi: 10.1097/WNR.0b013e32830e0025

Chib, V. S., Rangel, A., Shimojo, S., and O’Doherty, J. P. (2009). Evidence for a common representation of decision values for dissimilar goods in human ventromedial prefrontal cortex. J. Neurosci. 29, 12315-12320. doi: 10.1523/JNEUROSCI.2575-09.2009

Chong, T. T., Cunnington, R., Williams, M. A., Kanwisher, N., and Mattingley, J. B. (2008). fMRI adaptation reveals mirror neurons in human inferior parietal cortex. Curr. Biol. 18, 1576-1580. doi: 10.1016/j.cub.2008. 08.068

Cross, E. S., Kirsch, L., Ticini, L. F., and Schütz-Bosbach, S. (2011). The impact of aesthetic evaluation and physical ability on dance perception. Front. Hum. Neurosci. 5:102. doi: 10.3389/fnhum.2011.00102

Cross, E. S., and Ticini, L. F. (2012). Neuroaesthetics and beyond: new horizons in applying the science of the brain to the art of dance. Phenomenol. Cogn. Sci. 11, 5-16. doi: 10.1007/s11097-010-9190-y

D'Ausilio, A., Bufalari, I., Salmas, P., and Fadiga, L. (2012). The role of the motor system in discriminating normal and degraded speech sounds. Cortex 48, 882-887. doi: 10.1016/j.cortex.2011.05.017
Di Lazzaro, V., Pilato, F., Saturno, E., Oliviero, A., Dileone, M., Mazzone, P., et al. (2005). Theta-burst repetitive transcranial magnetic stimulation suppresses specific excitatory circuits in the human motor cortex. J. Physiol. 565, 945-950. doi: 10.1113/jphysiol.2005.087288

Dräger, B., Breitenstein, C., Helmke, U., Kamping, S., and Knecht, S. (2004). Specific and nonspecific effects of transcranial magnetic stimulation on picture-word verification. Eur. J. Neurosci. 20, 1681-1687. doi: 10.1111/j.1460-9568.2004.03623.x

Engelen, T., de Graaf, T. A., Sack, A. T., and de Gelder, B. (2015). A causal role for inferior parietal lobule in emotion body perception. Cortex 73, 195-202. doi: 10.1016/j.cortex.2015.08.013

Fabbri-Destro, M., and Rizzolatti, G. (2008). Mirror neurons and mirror systems in monkeys and humans. Physiology 23, 171-179. doi: 10.1152/physiol.00004.2008

Fernandino, L., and Iacoboni, M. (2010). Are cortical motor maps based on body parts or coordinated actions? Implications for embodied semantics. Brain Lang. 112, 44-53. doi: 10.1016/j.bandl.2009.02.003

Fitzgerald, P. B., Fountain, S., and Daskalakis, Z. J. (2006). A comprehensive review of the effects of rTMS on motor cortical excitability and inhibition. Clin. Neurophysiol. 117, 2584-2596. doi: 10.1016/j.clinph.2006.06.712

Fogassi, L., Ferrari, P. F., Gesierich, B., Rozzi, S., Chersi, F., and Rizzolatti, G. (2005). Parietal lobe: from action organization to intention understanding. Science 308, 662-667. doi: 10.1126/science.1106138

Fogassi, L., and Luppino, G. (2005). Motor functions of the parietal lobe. Curr. Opin. Neurobiol. 15, 626-631. doi: 10.1016/j.conb.2005.10.015

Franca, M., Koch, G., Mochizuki, H., Huang, Y. Z., and Rothwell, J. C. (2006). Effects of theta burst stimulation protocols on phosphene threshold. Clin. Neurophysiol. 117, 1808-1813. doi: 10.1016/j.clinph.2006.03.019

Freedberg, D., and Gallese, V. (2007). Motion, emotion and empathy in esthetic experience. Trends Cogn. Sci. 11, 197-203. doi: 10.1016/j.tics.2007.02.003

Friston, K., Mattout, J., and Kilner, J. (2011). Action understanding and active inference. Biol. Cybern. 104, 137-160. doi: 10.1007/s00422-011-0424-z

Gallese, V., Fadiga, L., Fogassi, L., and Rizzolatti, G. (1996). Action recognition in the premotor cortex. Brain 119(Pt 2), 593-609. doi: 10.1093/brain/119.2.593

Gazzola, V., and Keysers, C. (2009). The observation and execution of actions share motor and somatosensory voxels in all tested subjects: singlesubject analyses of unsmoothed fMRI data. Cereb. Cortex 19, 1239-1255. doi: $10.1093 /$ cercor/bhn181

Gentilucci, M., and Volta, R. D. (2008). Spoken language and arm gestures are controlled by the same motor control system. Q. J. Exp. Psychol. 61, 944-957. doi: 10.1080/17470210701625683

Girard, R. (1988). Violence and the Sacred. London: Athlone.

Gollwitzer, P. M., and Moskowitz, G. B. (1996). "Goal effects on action and cognition," in Social Psychology: Handbook of Basic Principles, eds E. T. Higgins and A. W. Kruglanski (NewYork, NY: Guilford), 361-399.

Goodale, M. A., and Milner, A. D. (1992). Separate visual pathways for perception and action. Trends Neurosci. 15, 20-25. doi: 10.1016/0166-2236(92)90344-8

Grafton, S. T. (2009). Embodied cognition and the simulation of action to understand others. Ann. N. Y. Acad. Sci. 1156, 97-117. doi: 10.1111/j.1749-6632.2009.04425.x

Grèzes, J., Armony, J. L., Rowe, J., and Passingham, R. E. (2003). Activations related to 'mirror' and 'canonical' neurones in the human brain: an fMRI study. Neuroimage 18, 928-937. doi: 10.1016/S1053-8119(03)00042-9

Huang, Y. Z., Edwards, M. J., Rounis, E., Bhatia, K. P., and Rothwell, J. C. (2005). Theta burst stimulation of the human motor cortex. Neuron 45, 201-206. doi: 10.1016/j.neuron.2004.12.033

Huang, Y. Z., and Rothwell, J. C. (2004). The effect of short-duration bursts of high-frequency, low-intensity transcranial magnetic stimulation on the human motor cortex. Clin. Neurophysiol. 115, 1069-1075. doi: 10.1016/j.clinph.2003.12.026

Iacoboni, M., Woods, R. P., Brass, M., Bekkering, H., Mazziotta, J. C., and Rizzolatti, G. (1999). Cortical mechanisms of human imitation. Science 286, 2526-2528. doi: 10.1126/science.286.5449.2526

Ishibashi, R., Ralph, M. A. L., Saito, S., and Pobric, G. (2011). Different roles of lateral anterior temporal lobe and inferior parietal lobule in coding function and manipulation tool knowledge: evidence from an rTMS study. Neuropsychologia 49, 1128-1135. doi: 10.1016/j.neuropsychologia.2011.01.004

Jacquet, P. O., and Avenanti, A. (2015). Perturbing the action observation network during perception and categorization of actions' goals and grips: 
state-dependency and virtual lesion TMS effects. Cereb. Cortex 25, 598-608. doi: $10.1093 /$ cercor/bht242

Jastorff, J., Begliomini, C., Fabbri-Destro, M., Rizzolatti, G., and Orban, G. A. (2010). Coding observed motor acts: different organizational principles in the parietal and premotor cortex of humans. J. Neurophysiol. 104, 128-140. doi: $10.1152 /$ jn.00254.2010

Jeannerod, M., Arbib, M. A., Rizzolatti, G., and Sakata, H. (1995). Grasping objects: the cortical mechanisms of visuomotor transformation. Trends Neurosci. 18, 314-320. doi: 10.1016/0166-2236(95)93921-J

Johnson-Frey, S. H., Maloof, F. R., Newman-Norlund, R., Farrer, C., Inati, S., and Grafton, S. T. (2003). Actions or hand-object interactions? Human inferior frontal cortex and action observation. Neuron 39, 1053-1058. doi: 10.1016/S0896-6273(03)00524-5

Kalénine, S., Buxbaum, L. J., and Coslett, H. B. (2010). Critical brain regions for action recognition: lesion symptom mapping in left hemisphere stroke. Brain 133, 3269-3280. doi: 10.1093/brain/awq210

Kellenbach, M. L., Brett, M., and Patterson, K. (2003). Actions speak louder than functions: the importance of manipulability and action in tool representation. J. Cogn. Neurosci. 15, 30-46. doi: 10.1162/089892903321107800

Kilner, J. M., Friston, K. J., and Frith, C. D. (2007). Predictive coding: an account of the mirror neuron system. Cogn. Process. 8, 159-166. doi: 10.1007/s10339-007-0170-2

Lebreton, M., Jorge, S., Michel, V., Thirion, B., and Pessiglione, M. (2009). An automatic valuation system in the human brain: evidence from functional neuroimaging. Neuron 64, 431-439. doi: 10.1016/j.neuron.2009.09.040

Lebreton, M., Kawa, S., Forgeot d'Arc, B., Daunizeau, J., and Pessiglione, M. (2012). Your goal is mine: unraveling mimetic desires in the human brain. J. Neurosci. 32, 7146-7157. doi: 10.1523/JNEUROSCI.4821-11.2012

Love, J., Selker, R., Marsman, M., Jamil, T., Verhagen, A. J., Ly, A., et al. (2015). JASP (Version 0.6.6) [Computer Software].

Molenberghs, P., Cunnington, R., and Mattingley, J. B. (2009). Is the mirror neuron system involved in imitation? A short review and meta-analysis. Neurosci. Biobehav. Rev. 33, 975-980. doi: 10.1016/j.neubiorev.2009.03.010

Mühlau, M., Hermsdörfer, J., Goldenberg, G., Wohlschläger, A. M., Castrop, F., Stahl, R., et al. (2005). Left inferior parietal dominance in gesture imitation: an fMRI study. Neuropsychologia 43, 1086-1098. doi: 10.1016/j.neuropsychologia.2004.10.004

Parkin, B. L., Ekhtiari, H., and Walsh, V. F. (2015). Non-invasive human brain stimulation in cognitive neuroscience: a primer. Neuron 87, 932-945. doi: 10.1016/j.neuron.2015.07.032

Pelphrey, K. A., Morris, J. P., and Mccarthy, G. (2004). Grasping the intentions of others: the perceived intentionality of an action influences activity in the superior temporal sulcus during social perception. J. Cogn. Neurosci. 16, 1706-1716. doi: 10.1162/0898929042947900

Peeters, R., Simone, L., Nelissen, K., Fabbri-Destro, M., Vanduffel, W., Rizzolatti, G., et al. (2009). The representation of tool use in humans and monkeys: common and uniquely human features. J. Neurosci. 29, 11523-11539. doi: 10.1523/JNEUROSCI.2040-09.2009

Peters, J., and Büchel, C. (2010). Neural representations of subjective reward value. Behav. Brain Res. 213, 135-141. doi: 10.1016/j.bbr.2010.04.031

Ping, R. M., Dhillon, S., and Beilock, S. L. (2009). Reach for what you like: the body's role in shaping preferences. Emot. Rev. 1, 140-150. doi: 10.1177/1754073908100439

Proverbio, A., Del Zotto, M., and Zani, A. (2007). The emergence of semantic categorization in early visual processing: ERP indices of animal vs. artifact recognition. BMC Neurosci. 8:24. doi: 10.1186/1471-2202-8-24

Proverbio, A. M., Adorni, R., and D'Aniello, G. E. (2011). $250 \mathrm{~ms}$ to code for action affordance during observation of manipulable objects. Neuropsychologia 9, 2711-2717. doi: 10.1016/j.neuropsychologia.2011. 05.019

Proverbio, A. M., Azzari, R., and Adorni, R. (2013). Is there a left hemispheric asymmetry for tool affordance processing. Neuropsychologia 51, 2690-2701. doi: 10.1016/j.neuropsychologia.2013.09.023
Puzzo, I., Cooper, N. R., Cantarella, S., Fitzgerald, P. B., and Russo, R. (2013). The effect of rTMS over the inferior parietal lobule on EEG sensorimotor reactivity differs according to self-reported traits of autism in typically developing individuals. Brain Res. 1541, 33-41. doi: 10.1016/j.brainres.2013.10.016

Rangel, A., Camerer, C., and Montague, P. R. (2008). A framework for studying the neurobiology of value-based decision making. Nat. Rev. Neurosci. 9, 545-556. doi: $10.1038 / \mathrm{nrn} 2357$

Reber, R., Schwarz, N., and Winkielman, P. (2004). Processing fluency and aesthetic pleasure: is beauty in the perceiver's processing experience? Pers. Soc. Psychol. Rev. 8, 364-382. doi: 10.1207/s15327957pspr0804_3

Rizzolatti, G., and Craighero, L. (2004). The mirror-neuron system. Annu. Rev. Neurosci. 27, 169-192. doi: 10.1146/annurev.neuro.27.070203.144230

Rosci, C., Chiesa, V., Laiacona, M., and Capitani, E. (2003). Apraxia is not associated to a disproportionate naming impairment for manipulable objects. Brain Cogn. 53, 412-415. doi: 10.1016/S0278-2626(03)00156-8

Rouder, J. N., Morey, R. D., Speckman, P. L., and Province, J. M. (2012). Default Bayes factors for ANOVA designs. J. Math. Psychol. 56, 356-374. doi: 10.1016/j.jmp.2012.08.001

Sack, A. T., Kadosh, R. C., Schuhmann, T., Moerel, M., Walsh, V., and Goebel, R. (2009). Optimizing functional accuracy of TMS in cognitive studies: a comparison of methods. J. Cogn. Neurosci. 21, 207-221. doi: 10.1162/jocn.2009.21126

Schippers, M. B., and Keysers, C. (2011). Mapping the flow of information within the putative mirror neuron system during gesture observation. Neuroimage 57, 37-44. doi: 10.1016/j.neuroimage.2011.02.018

Shmuelof, L., and Zohary, E. (2006). A mirror representation of others' actions in the human anterior parietal cortex. J. Neurosci. 26, 9736-9742. doi: 10.1523/JNEUROSCI.1836-06.2006

Siebner, H. R., Takano, B., Peinemann, A., Schwaiger, M., Conrad, B., and Drzezga, A. (2001). Continuous transcranial magnetic stimulation during positron emission tomography: a suitable tool for imaging regional excitability of the human cortex. Neuroimage 14, 883-890. doi: 10.1006/nimg.20 01.0889

Silvanto, J., Cattaneo, Z., Battelli, L., and Pascual-Leone, A. (2008). Baseline cortical excitability determines whether TMS disrupts or facilitates behavior. J. Neurophysiol. 99, 2725-2730. doi: 10.1152/jn.01392.2007

Stewart, L. M., Walsh, V., and Rothwell, J. C. (2001). Motor and phosphene thresholds: a transcranial magnetic stimulation correlation study. Neuropsychologia 39, 415-419. doi: 10.1016/S0028-3932(00)00130-5

Ticini, L. F., Rachman, L., Pelletier, J., and Dubal, S. (2014). Enhancing aesthetic appreciation by priming canvases with actions that match the artist's painting style. Front. Hum. Neurosci. 8:391. doi: 10.3389/fnhum.2014.00391

Ubaldi, S., Barchiesi, G., and Cattaneo, L. (2015). Bottom-up and top-down visuomotor responses to action observation. Cereb. Cortex 25, 1032-1041. doi: 10.1093/cercor/bht295

Urgesi, C., Candidi, M., and Avenanti, A. (2014). Neuroanatomical substrates of action perception and understanding: an anatomic likelihood estimation metaanalysis of lesion-symptom mapping studies in brain injured patients. Front. Hum. Neurosci. 8:344. doi: 10.3389/fnhum.2014.00344

Wetzels, R., and Wagenmakers, E. J. (2012). A default Bayesian hypothesis test for correlations and partial correlations. Psychon. Bull. Rev. 19, 1057-1064. doi: 10.3758/s13423-012-0295-x

Conflict of Interest Statement: The authors declare that the research was conducted in the absence of any commercial or financial relationships that could be construed as a potential conflict of interest.

Copyright (c) 2017 Ticini, Urgesi and Kotz. This is an open-access article distributed under the terms of the Creative Commons Attribution License (CC BY). The use, distribution or reproduction in other forums is permitted, provided the original author(s) or licensor are credited and that the original publication in this journal is cited, in accordance with accepted academic practice. No use, distribution or reproduction is permitted which does not comply with these terms. 